\title{
Toward the integration of personalized and systems medicine: challenges, opportunities and approaches
}

\author{
"The integration of personalized and systems medicine offers the prospect of the \\ development of a better medicine that provides optimal therapies with high \\ efficacy and low adverse drug reactions."
}

\section{KEYWORDS: biomarkers $\approx$ genotypes $\approx$ networks $\approx$ pathways $\approx$ personalized medicine - pharmacogenomics " phenotypes " systems biology $\approx$ translational bioinformatics - wellness}

\section{Challenges \& new concepts in biomedicine}

In this era of change, biomedicine is heading toward a revolutionary new path. This path is a transformation from reductionism toward a holistic paradigm, from 'one-size-fits-all' therapeutics towards personalized medicine. Such changes are necessary to meet the challenges in healthcare and the pharmaceutical industry, for example, the high costs in healthcare, low efficacy of drugs and increased incidents of adverse drug reactions (ADRs). ADRs are one of the leading causes of death and illness in the USA [1]. Although the research and development costs in the pharmaceutical industry are soaring, high-profile drug withdrawals are elevating, while the US FDA's approval for new drugs is decreasing [2]. At the same time, the gap between biomedical science and clinical practice has made it hard to translate scientific advancements into improved healthcare.

These challenges and difficulties are calling for the development of new strategies, new concepts, or even a new kind of medicine to meet these goals:

- Providing individualized optimal therapy with high efficacy and low ADRs;

- Treatment of the whole system with a focus on prevention of disease;

- Promotion of the mind-body health and wellness in the physical, mental, social and environmental dimensions [3].

Such 'new medicine' will come from the integration of personalized and systems medicine. Ideally, the new medicine will bring the right prevention methods or therapeutics to the right person or patient with the right dosages at the right time.

\section{Personalized medicine: from disease-centered to human-centered medicine}

Personalized medicine deals with individual diversities and the complexity of the human body. Current healthcare models are diseaseoriented and focus on pathogens and environmental or external factors [4]. Personalized medicine would enable the change from such disease-centered medicine to human-centered medicine. Pharmacogenomics arose in response to such recognition. Pharmacogenomics studies genetic variations among individuals to predict disease susceptibility and responses to therapeutic agents $[5,6]$. The investigation of genetic diversity may enable the identification of optimal drug targets for certain patient populations, and empower physicians to make the right decisions. By focusing on patients' genetic or biomarker profiles, pharmacogenomics represents the evolution from treating the disease itself to treating the malfunction of an individual person, the 'root' of the disease [7]. With such practices, patients can be regrouped and drugs can be recategorized. For instance, similar diseases may require different treatments, while different diseases may be treated with the same or similar approaches.

Because of the diversity of patients' biological backgrounds, the same disease may be caused by genetic variation in different people, who will respond differently to the same drug. For example, polymorphisms in the $A B C B 1$ gene contribute to cancer risk and different therapeutic responses [8]. Patients with certain genotypes, such as $1236 \mathrm{C} / \mathrm{C}$, showed poorer survival than patients with other genotypes. Such situations require individualized treatments for the same disease (cancer) to ensure the best possible results. On the other hand,

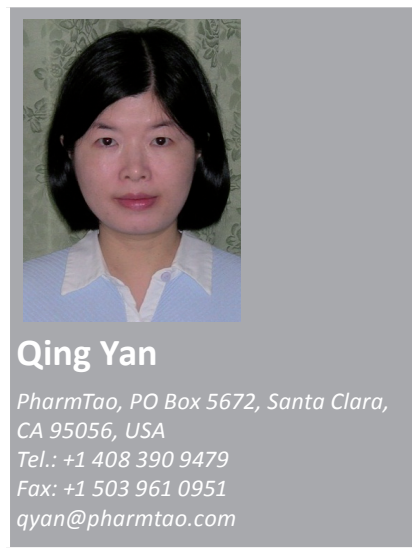

future micine $^{\text {pet }}$ fsg 
patients with the same or similar biomarker profiles but with different diseases, may be grouped together to accept the same or similar therapeutics. For example, inflammation is a multiscale process associated with many diseases from cardiovascular disease to cancer [9]. The same genetic factors involved in inflammation, such as peroxisome proliferator activated receptor $\gamma(P P A R \gamma)$, can serve as potent drug targets for different diseases, including diabetes, allergic diseases and respiratory virus infections $[10,11]$.

\section{"This new medicine would be human-centric with the focus on prevention and the promotion of holistic health in the physical, mental and environmental dimensions."}

Such an approach of regrouping of patients and recategorization of drugs based on patients' biomarker profiles (but not diseases) would have many benefits. It may help reduce the adverse events and improve the therapeutic efficacy when the drugs can match the patients seamlessly. In addition, the application range of existing drugs may be broadened to more diseases, and drug combination therapies can be used, which would actually save money for the healthcare industry. More importantly, this approach would bring hope for the transformation from disease treatment to prevention. The biomarker profiling of patients may allow for the prediction of disease predispositions and prognosis. Such a type of prediction would enable the prevention of the diseases and block the disease development path for early-stage cures. This is how pharmacogenomics will contribute to preventive medicine and help solve the problems of high costs, low efficacy and high ADRs in healthcare.

\section{Integration with systems medicine: understanding multidimensional interactions}

While pharmacogenomics helps elucidate the association between patient genetic diversity and phenotypic responses, such studies should not be limited to single biomarker genes or SNPs [12]. This is because genes interact with each other, as well as with other molecules such as drugs and the environment. Using computational methods, systems biology may help us simulate large networks of interacting components, identify associations and patterns, and create predictive models [13]. Systems medicine, the practice of systems biology, needs to be integrated into the drug-development processes to solve the key issues in pharmacogenomics. These key issues include the structure-function relationships at various levels, the genes-drugs-environment interactions and the association of genotypes to disease and drug-response phenotypes [13].

These key issues are correlated with each other at the molecular, cellular, tissue/organ, system and environmental levels. Specifically, altered genetic structures may cause malfunctions at the molecular level and affect the downstream pathways and interactions among different components at the cellular level [13]. These alterations may lead to tissue or organ disorders that are manifested as disease phenotypes and symptoms of the whole body. Furthermore, altered genetic structures and functions may affect the gene-drug interactions and drug-response phenotypes. On the other hand, interactions among systems, drugs and the environment at higher levels may have an impact on genetic structures and functions at the molecular level, which would, in turn, change downstream pathways and phenotypes, forming a feedback loop. The understanding of such an interwoven network may be the ultimate key to accurately identifying drug targets and to preventing adverse reactions.

\section{"...the application of pharmacogenomics and systems biology will bring new concepts and methods into medicine, such as the regrouping of patients and recategorization of drugs based on pattern identifications and systemic biomarker profiles."}

Among these issues, the genotype-phenotype correlation connects the information at different dimensions from the molecular level to the whole organism. This correlation represents the core value of systems biology, demonstrating that the behavior of a whole system does not merely come from its separated building blocks, but through the complex interrelationships and interactions between them [12]. A good example of this is inflammation, one of the best models for applying the translational systems biology framework [9,14]. For instance, complex NF- $\kappa \mathrm{B}$ and p38 signaling pathways are involved in various phenotypes of inflammation, cancer and microbial infections [14]. The exploration of systemic interactions and feedbacks rather than single molecules is needed to understand the inflammatory processes and relevant disease phenotypes. 
Systems medicine would also contribute to the understanding of the mind-body relationships for achieving the ultimate goal of wellness in the physiological and mental dimensions. Emerging multidisciplinary areas such as psychoneuroimmunology (PNI) may benefit greatly from systems biology approaches. PNI studies the correlations and interactions among the psychological processes, the nervous system, the immune system and the endocrine system [15]. For example, the cytokine network plays a central role in PNI $[16,17]$. The brain cytokines, including $I L-1 \beta$ and $I L-6$, are involved in food intake, fever, sickness and anxiety-like behaviors $[18,19]$. Systems biology approaches can help construct computational models of such highly intercorrelated and multivariate networks in mind-body connections [17].

\section{Tools for the practice: \\ biomarkers, technologies \& translational bioinformatics}

An important component in the accurate practice of personalized and systems medicine is systemic biomarker identification, which is crucial for quantified diagnosis and prognosis, treatment selection and the profiling and grouping of patients. Biomarkers are objectively measured indicators of biologic states such as diseases and drug responses [12]. They can be analyzed in various functional pathways at different systems levels, including genetic markers at the molecular level, and imaging and physiological indicators at the system level [12]. Multiple biomarkers need to be combined in a comprehensive profile that summarizes the diagnostic and drug-response patterns which can then be used to identify patient subgroups and establish predictive models. For example, predictive and prognostic biomarkers for different subsets of patients have been used for outcome prediction and assessment in diseases including cancer, cardiovascular diseases, respiratory diseases and neurological diseases [12] .

Advanced technologies and tools are crucial for such comprehensive biomarker identifications. High-throughput technologies enable the measurement and cataloging of genes, proteins, interactions and behaviors in various conditions [12] For instance, protein microarrays have been used extensively for pathways and pharmacoproteomics studies [20]. These methods allow genomewide association studies to identify biomarkers for diagnosis and outcome assessments [14]. For example, genome-wide association studies have identified distinct cancer susceptibility loci among different ethnic groups [21].
One of the most significant obstacles in current medical practice is the translation of scientific discoveries into better therapeutic outcomes. A critical factor in this process is the accessibility and analysis of integrated data within and across functional domains [12]. Translational bioinformatics is a powerful method to bridge this gap from the biomedical side to the informatics side [12]. On the biomedical side, translational bioinformatics would enable the simulation of networks of interacting components, the identification of patterns and patient subgroups from biomarker profiles and the establishment of predictive models. For example, a bioinformatics tool was used to correlate biomarkers including HER2 and estrogen receptor with survival outcomes in breast cancer patients [22]. The tool identified two distinct subpopulations of tumors with different levels of expressers. Moreover, on the informatics side, methods based on data integration, data mining and knowledge representation can promote data sharing across domains and provide decision support for both researchers and clinicians [12]. For example, the integration of information from multiple layers of biological regulation from multiple genome-wide data sources has been found to improve the prediction of cancer outcome [23].

\section{Conclusion \& future perspective}

Although biomedicine is facing many obstacles and difficulties, appropriate solutions can transform the challenges into opportunities. The integration of personalized and systems medicine offers the prospect of the development of a better medicine that provides optimal therapies with high efficacy and low ADRs. This new medicine would be human-centric with the focus on prevention and the promotion of holistic health in the physical, mental and environmental dimensions. Specifically, the application of pharmacogenomics and systems biology will bring new concepts and methods into medicine, such as the regrouping of patients and recategorization of drugs based on pattern identifications and systemic biomarker profiles. These approaches would enable the construction of predictive models for the understanding of structure-function relationships, genotype-phenotype associations and interactions and networks at the molecular, cellular, system and environmental levels [13]. These practices would also improve our understanding of the mind-body relationships toward achieving the ultimate goal of personalized wellness. Tools such as high-throughput technologies and translational bioinformatics would serve as the technologies to bring us to this bright future. 
Financial \& competing interests disclosure The author has no relevant affiliations or financial involvement with any organization or entity with a financial interest in or financial conflict with the subject matter or materials discussed in the manuscript. This includes employment, consultancies, honoraria, stock ownership or options, expert testimony, grants or patents received or pending, or royalties.

No writing assistance was utilized in the production of this manuscript.

\section{Bibliography}

1 Ross CJ, Carleton B, Warn DG et al.: Genotypic approaches to therapy in children: a national active surveillance network (GATC) to study the pharmacogenomics of severe adverse drug reactions in children. Ann. NY Acad. Sci. 1110, 177-192 (2007).

2 Caskey CT: The drug development crisis: efficiency and safety. Annu. Rev. Med. 58, 1-16 (2007).

3 Hawks SR, Smith T, Thomas HG et al:: The forgotten dimensions in health education research. Health Educ. Res. 23(2), 319-324 (2008).

4 Ray O: The revolutionary health science of psychoendoneuroimmunology: a new paradigm for understanding health and treating illness. Ann. NY Acad. Sci. 1032, 35-51 (2004).

5 Yan Q: Pharmacogenomics of membrane transporters an overview. Methods Mol. Biol. 227, 1-20 (2003).

6 Meyer UA: Pharmacogenetics - five decades of therapeutic lessons from genetic diversity. Nat. Rev. Genet. 5, 669-676 (2004).

7 Yan Q: Pharmacogenomics in drug discovery and development. Preface. Methods Mol. Biol. 448, v-vii (2008).

8 Gréen H, Falk IJ, Lotfi K et al.: Association of $A B C B 1$ polymorphisms with survival and in vitro cytotoxicty in de novo acute myeloid leukemia with normal karyotype. Pharmacogenomics J. (2010) (Epub ahead of print).
9 Vodovotz Y, An G: Systems biology and inflammation. Methods Mol. Biol. 662, 181-201 (2010).

10 Martin H: Role of PPAR- $\gamma$ in inflammation. Prospects for therapeutic intervention by food components. Mutat. Res. 690(1-2), 57-63 (2010).

11 Bassaganya-Riera J, Song R, Roberts PC et al.: PPAR- $\gamma$ activation as an anti-inflammatory therapy for respiratory virus infections. Viral Immunol. 23(4), 343-352 (2010).

12 Yan Q: Translational bioinformatics and systems biology approaches for personalized medicine. Methods Mol. Biol. 662, 167-178 (2010).

13 Yan Q: The integration of personalized and systems medicine: bioinformatics support for pharmacogenomics and drug discovery. Methods Mol. Biol. 448, 1-19 (2008).

14 Yan Q: Immunoinformatics and systems biology methods for personalized medicine. Methods Mol. Biol. 662, 203-220 (2010).

15 Godbout JP, Glaser R: Stress-induced immune dysregulation: implications for wound healing, infectious disease and cancer. J. Neuroimmune Pharmacol. 1(4), 421-427 (2006).

16 Marques-Deak A, Sternberg E: Psychoneuroimmunology - the relation between the central nervous system and the immune system. Rev. Bras. Psiquiatr. 26(3), 143-144 (2004).

17 Goncharova LB, Tarakanov AO: Molecular networks of brain and immunity. Brain Res. Rev. 55(1), 155-166 (2007).
18 Skurlova M, Stofkova A, Jurcovicova J: Anxiety-like behavior in the elevated-plus maze tests and enhanced IL- $1 \beta$, IL-6, NADPH oxidase-1, and iNOS mRNAs in the hippocampus during early stage of adjuvant arthritis in rats. Neurosci. Lett. (2010) (Epub ahead of print)

19 Harden LM, du Plessis I, Roth J et al.: Differences in the relative involvement of peripherally released interleukin (IL)-6, brain IL- $1 \beta$ and prostanoids in mediating lipopolysaccharide-induced fever and sickness behavior. Psychoneuroendocrinology (2010) (Epub ahead of print).

20 Wulfkuhle JD, Edmiston KH, Liotta LA et al.: Technology insight:

pharmacoproteomics for cancer - promises of patient-tailored medicine using protein microarrays. Nat. Clin. Pract. Oncol. 3(5), 256-268 (2006).

21 Chung CC, Magalhaes WC, Gonzalez-Bosquet J et al.: Genome-wide association studies in cancer - current and future directions. Carcinogenesis 31(1), 111-120 (2010).

22 Camp RL, Dolled-Filhart M et al.: X-tile: a new bioinformatics tool for biomarker assessment and outcome-based cutpoint optimization. Clin. Cancer Res. 10, 7252-7259 (2004).

23 Daemen A, Gevaert O, Ojeda F et al.: A kernel-based integration of genome-wide data for clinical decision support. Genome Med. 1, 39 (2009). 